\title{
Kitaev magnetism and fractionalized excitations in double perovskite $\mathrm{Sm}_{2} \mathrm{ZnIrO}_{6}$
}

\author{
Birender Singh $\odot,{ }^{1, *}$ M. Vogl, ${ }^{2}$ S. Wurmehl, ${ }^{2,3}$ S. Aswartham, ${ }^{2}$ B. Büchner, ${ }^{2,3}$ and Pradeep Kumar ${ }^{1, \dagger}$ \\ ${ }^{1}$ School of Basic Sciences, Indian Institute of Technology Mandi, Mandi 175005, India \\ ${ }^{2}$ Leibniz Institute for Solid State and Materials Research, IFW-Dresden, 01069 Dresden, Germany \\ ${ }^{3}$ Institute of Solid State Physics, TU Dresden, 01069 Dresden, Germany
}

(Received 9 September 2019; revised manuscript received 29 October 2019; published 13 January 2020)

\begin{abstract}
The quest for Kitaev spin liquids in particular three-dimensional solids is a long sought goal in condensed matter physics, as these states may give rise to exotic new types of quasiparticle excitations carrying fractional quantum numbers, namely Majorana fermionic excitations. Here we report the experimental signature of this characteristic feature of the Kitaev spin liquid via Raman measurements. $\mathrm{Sm}_{2} \mathrm{ZnIrO}_{6}$ is a strongly spin-orbit-coupled Mott insulator where $J_{\text {eff }}=1 / 2$ controls the physics, which provides striking evidence for this characteristic feature of the Kitaev spin liquid. As the temperature is lowered, we find that the spin excitations form a continuum in contrast to the conventional sharp modes expected in ordered antiferromagnets. Our observation of a broad magnetic continuum and anomalous renormalization of the phonon self-energy parameters shows the existence of fractionalization excitations in the double-perovskite structure, as theoretically conjectured in a Kitaev-Heisenberg geometrically frustrated double-perovskite system.
\end{abstract}

DOI: 10.1103/PhysRevResearch.2.013040

\section{INTRODUCTION}

Quantum magnetic materials having partially filled $5 d$ elements are the subject of extensive studies, as these are predicted to host exotic quantum phases. These materials open up a new avenue in the field of condensed matter physics due to the presence of interesting competing interactions of similar energy scale, such as the on-site Coulomb interaction, strong spin-orbit (SO) coupling, Hund's coupling, and crystal-field splitting, involving the interplay of spin, orbital, charge, and lattice degrees of freedom [1-5]. Fierce competition between different degrees of freedom provides fertile ground to observe rich magnetic ground states such as the quantum spin liquid (QSL). The experimental realization of the QSL, a state which precludes long-range ordering of spins even at absolute-zero temperature, is a long sought goal in physics, as it represents new states of matter. Systems with the QSL as their ground state $(|\mathrm{GS}\rangle)$ are anticipated to be topologically active and to host exotic low-energy quasiparticle excitations such as Majorana fermions, which result from spin fractionalization, and gauge fields.

The so-called Kitaev Hamiltonian, in which the nearestneighbor Ising-type interactions are bond-directional [6], is an exactly solvable model with QSL as the $|\mathrm{GS}\rangle$. The recent search is for systems which realize these exotic states. As one example, it was proposed [7] that the strong SO-coupled

\footnotetext{
*birender.physics5390@gmail.com

†pkumar@iitmandi.ac.in
}

Published by the American Physical Society under the terms of the Creative Commons Attribution 4.0 International license. Further distribution of this work must maintain attribution to the author(s) and the published article's title, journal citation, and DOI.
Mott insulators with an edge-sharing octahedral environment may give rise to a QSL as the ground state. To date very few systems, such as the $2 \mathrm{D}$ honeycomb based structure $A_{2} \mathrm{IrO}_{3}(A=\mathrm{Na}, \mathrm{Li})$ and $\mathrm{RuCl}_{3}$ [8-10], fulfil this criterion. However, the elusive Kitaev spin liquid state in these systems is preempted by the long-range magnetic order at low temperature possibly resulting from the presence of interaction beyond the Kitaev model such as Heisenberg off-diagonal interactions. Interestingly, in spite of long-range magnetic ordering, the existence of spin fractionalization and Majorana fermionic type excitations in these systems was inferred from the light-scattering experiments via the observation of a broad continuum in Raman and inelastic neutron scattering [11-13] measurements, in contrast to the sharp magnon modes observed in typical long-range magnetically ordered systems [14-15], suggesting that the ordered $|\mathrm{GS}\rangle$ may be proximate to a quantum phase transition into the Kitaev spin liquid $|G S\rangle$. The hunt is on to find experimental evidence of a true Kitaev spin liquid especially in 3D systems. Recently, it has been suggested theoretically that strong SO-coupled Mott insulators controlled by $J_{\text {eff }}=1 / 2$ physics such as found in iridium-based double perovskites (DPs) $A_{2} B \operatorname{IrO}_{6}(A=$ $\mathrm{La}, B=\mathrm{Zn}, \mathrm{Mg}$ ) support symmetry-allowed nearest-neighbor interactions such as the Heisenberg and Kitaev interactions and symmetric off-diagonal exchange [16-17]. It is advocated that these 3D geometrically frustrated DP systems support Kitaev interactions as the dominating exchange along with the Heisenberg interactions with the $A$-type antiferromagnetic state as their $|\mathrm{GS}\rangle$, with a total Hamiltonian given as $H=$ $\sum_{i, j}\left(J_{K} S_{i}^{\mu} S_{j}^{\mu}+J \vec{S}_{i} \cdot \vec{S}_{j}\right)$ [17]. Here $J_{K}$ and $J$ are the Kitaev and Heisenberg exchange parameters, respectively; $\mu$ is the component of the spin directed perpendicularly to the bond connecting spins $(i, j)$, and the pure Kitaev spin liquid phase $(J=0)$ is stable against the perturbative Heisenberg interactions. Recent neutron diffraction measurements also confirm 

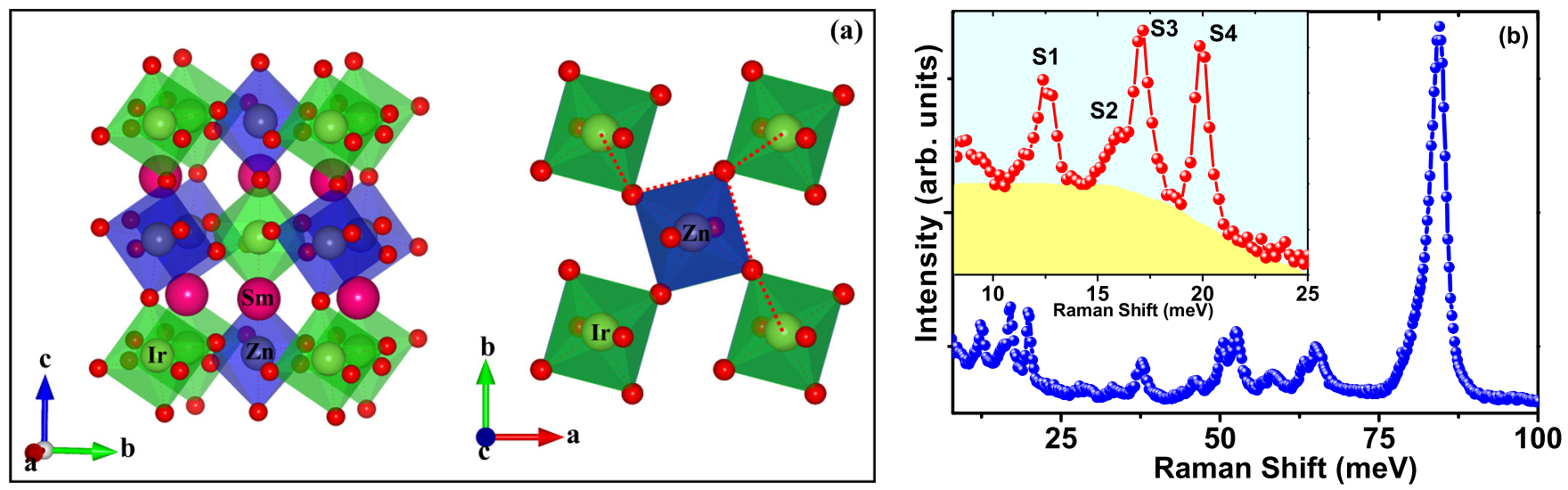

FIG. 1. Crystal structure and Raman spectra. (a) Schematic of the crystal structure of the double perovskite $\mathrm{Sm}_{2} \mathrm{ZnIrO}_{6}$ (pink, gray, dark yellow, and red spheres represent $\mathrm{Sm}, \mathrm{Zn}$, Ir, and oxygen atoms, respectively). Schematic on the right side of the panel shows the $a b$ plane indicating Ir-O-O-Ir exchange paths with dotted red lines. Green and blue depict $\mathrm{Ir}$ and $\mathrm{ZnO}_{6}$ octahedra, respectively. (b) Raman spectra of $\mathrm{Sm}_{2} \mathrm{ZnIrO}_{6}$ at $4 \mathrm{~K}$ in the energy range of 7-100 meV showing only first-order phonon modes. Inset shows the zoomed-in view of the low-energy spectrum below $25 \mathrm{meV}$; yellow shading is a guide to the eye and indicates the underlying continuum at $4 \mathrm{~K}$, superimposed on the distinct low-energy phonon modes.

this $A$-type antiferromagnetic state in $\mathrm{La}_{2} B \mathrm{IrO}_{6}$ and $A_{2} \mathrm{CeIrO}_{6}$ ( $A=\mathrm{Ba}, \mathrm{Sr} ; B=\mathrm{Zn}, \mathrm{Mg}$ ), thus supporting the existence of strong Kitaev interactions in these systems [18-21] owing to almost cancellation of the Heisenberg interactions from multiple Ir-O-O-Ir superexchange paths. It is also suggested that in these systems even long-range magnetic order may be driven by highly directional exchange interactions owing to strong SO coupling and strong magnetoelastic effects.

Motivated by these exciting proposals of a possible Kitaev quantum spin liquid phase in these strong SO-coupled Mott insulators, we took up inelastic light scattering (Raman) studies to probe the quasiparticle excitations in DP $\mathrm{Sm}_{2} \mathrm{ZnIrO}_{6}$, with $\operatorname{Ir}^{4+}\left(5 d^{5}\right)$, where the physics is governed by the $J_{\text {eff }}=$ $1 / 2$ picture. Unlike in the case of the $2 \mathrm{D}$ honeycomb structure these systems do not feature direct edge sharing $\mathrm{IrO}_{6}$ octahedra though they do have varying Ir-O-O-Ir exchange paths, and these multiple exchange paths may easily support oxygenmediated electron hopping leading to significant Kitaev interactions [19-20]. The smoking gun evidence for a Kitaev kind of anisotropic coupling is hidden in the quantum spin fluctuations and may reveal itself indirectly via interacting with the photon and coupling with the underlying magnetic spectrum. Raman scattering directly probes the Majorana fermion density of states as the magnetic Raman intensity of a Kitaev system is $\propto \sum_{i, j, q}\left(\omega-\varepsilon_{i, q}-\varepsilon_{j, q}\right)\left|M_{i, j, q}\right|^{2}$, where $\varepsilon_{i, j ; q}$ is the Majorana spinon dispersion and $M_{i, j, q}$ is the matrix element creating Majorana excitations [22-23]. Raman scattering is a very powerful probe to capture the signatures of these exotic quasiparticle excitations as it can simultaneously probe charge, spin, lattice, orbital, and electronic excitations [24-27].

\section{RESULTS AND DISCUSSION}

The double perovskite $\mathrm{Sm}_{2} \mathrm{ZnIrO}_{6}$ crystallizes in the monoclinic structure $\left(P 2_{1} / n\right.$; space group No. 14) and consists of corner-sharing $\mathrm{IrO}_{6}$ octahedra [see Fig. 1(a)]; magnetic measurements suggest an AFM ordering at $T_{N} \sim 13 \mathrm{~K}$ and tiny magnetic moments [28]. To our knowledge there has been so far no report on this $3 \mathrm{D}$ double-perovskite system probing spin excitations and exploring the possibility of anticipated spin fractionalization driven by strong SO coupling, a necessary denominator of the Kitaev spin liquid $|G S\rangle$. Our measurements reveal a broad anomalous magnetic continuum, unlike sharp features which are a hallmark of the long-range magnetic ordering, that persists in the magnetically ordered state below $13 \mathrm{~K}$. This anomalous evolution cannot be captured by the conventional two-magnon scattering; however, it seems consistent with the theoretical predictions for the spin-liquid phase. Figure 1(b) shows the Raman spectra at $4 \mathrm{~K}$ showing only the first-order modes. A broad continuum with spectral weight at low energy survived till $\sim 25 \mathrm{meV}$ [see Fig. 1(b) inset, marked by the yellow shading], and is attributed to the underlying magnetic scattering. To rule out the luminescence origin of this background, Raman measurements were carried out using two different wavelengths (see inset of Fig. 5 of the Appendix) of $\lambda=532 \mathrm{~nm}(2.33 \mathrm{eV})$ and $633 \mathrm{~nm}(1.96 \mathrm{eV})$. Detailed analysis of the first as well as second order phonon modes is given in the Appendix, along with the first-principles density functional theory (DFT) based calculations of the phonon dispersion.

Optical phonons may provide crucial information on the underlying magnetic continuum via strong coupling between these two degrees of freedom. The asymmetric line shape of a mode near $\sim 20 \mathrm{meV}$ [S4; see Fig. 1(b) inset] is a clear indication of a Fano resonance and reflects the coupling of this mode with a broad continuum of magnetic origin. For a quantitative analysis, this mode is fitted to a Fano profile [29], $F(\omega)=$ $I_{0}(q+\varepsilon)^{2} /\left(1+\varepsilon^{2}\right)$, where $\varepsilon=\left(\omega-\omega^{\prime}\right) / \Gamma ; \omega^{\prime}$ and $\Gamma$ are the phonon frequency and linewidth, respectively, and $q$ defines the nature of asymmetry.

Figures 2(a)-2(c) display the temperature dependence of the mode frequency $\left(\omega^{\prime}\right)$ and linewidth $(\Gamma)$ of the phonon $\mathrm{S} 4(\sim 20 \mathrm{meV})$, a schematic representation of the eigenvectors for the S4 mode, and the Fano asymmetry parameter $(1 /|q|)$. For the purpose of comparison, we have also plotted the self-energy parameter for the strongest mode S16 in the bottom panel of Fig. 2(a). The temperature dependence of the mode frequency and linewidth may be captured by the 

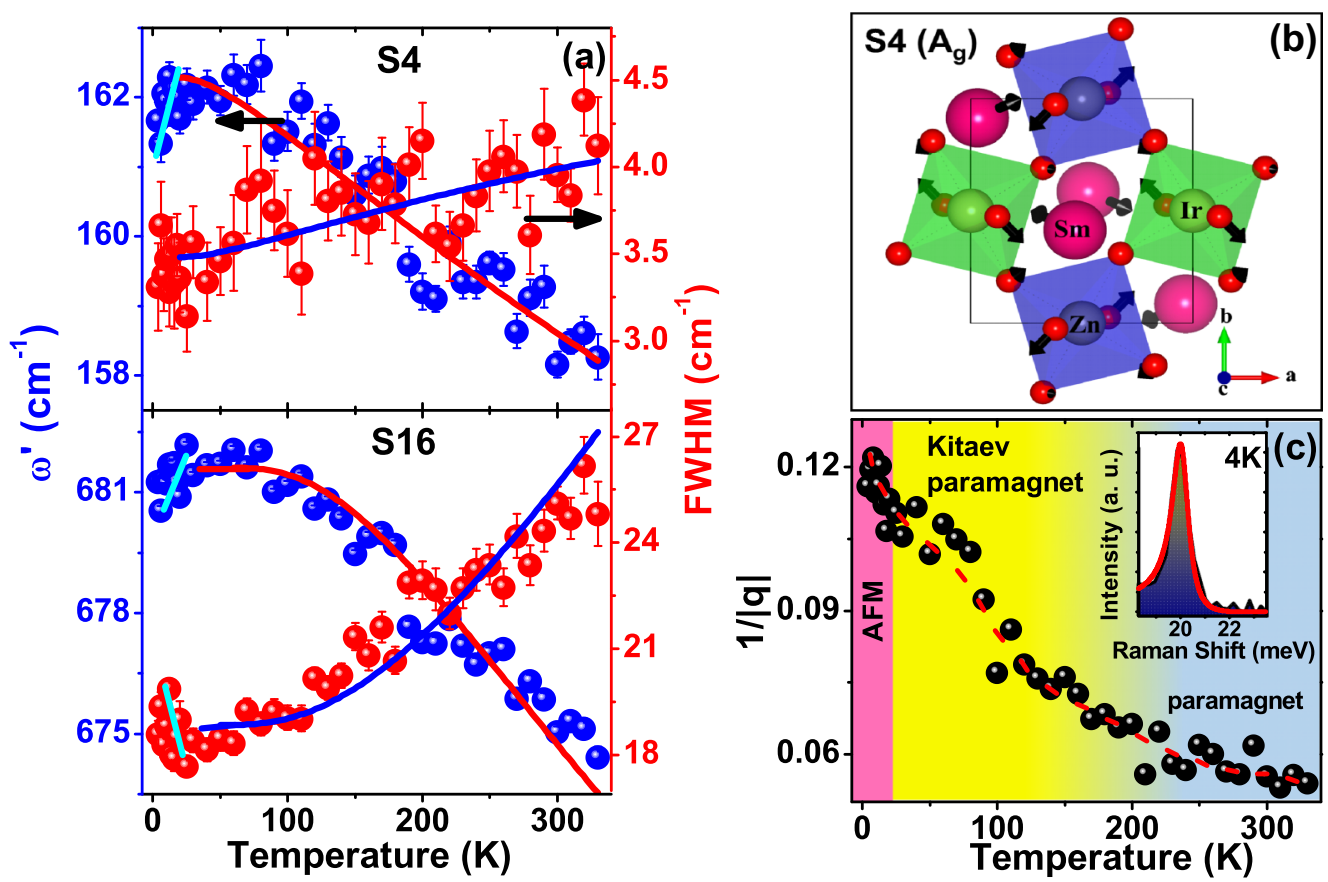

FIG. 2. Fano resonance and spin-phonon coupling. (a) Temperature variation of the self-energy parameters, i.e., mode frequency and linewidth, of the mode S4 ( 20 meV) and the strongest mode S16 ( $\sim 5 \mathrm{meV})$. (b) Schematic representation of the eigenvectors for the S4 mode; the amplitude and direction of the vibration are represented by the arrow length and head, respectively. Pink, gray, dark yellow, and red spheres represent $\mathrm{Sm}, \mathrm{Zn}$, Ir, and oxygen atoms, respectively. Sm and oxygen atoms exhibit out-of-phase motion, modulating the bonds mediating the complex exchange interactions. (c) Temperature dependence of the Fano asymmetry parameter $1 /|q|$ (dotted red line is a guide to the eye). Inset: The asymmetry in line shape at $4 \mathrm{~K}$ (solid red line is the fitted line with the Fano function and black shows the raw spectra). Different colored background shading in panel (c) reflects the different magnetic phases.

anharmonic model (see the Appendix for details) above $T_{N}$ $(\sim 13 \mathrm{~K})$, and below $T_{N}$ it shows softening attributed to the spin-phonon coupling in the long-range magnetically ordered phase. Interestingly, the temperature variation of $\omega^{\prime}$ shows a slight jump around $\sim 160-180 \mathrm{~K}$, and a similar jump in the frequency is also seen for other prominent modes (see Fig. 8 of the Appendix), signaling a subtle local structural change near this temperature. The Fano asymmetry parameter $(1 /|q|)$ characterizes the coupling strength of a phonon to the underlying continuum: a stronger coupling $(q \rightarrow 0)$ causes the peak to be more asymmetric and in the weak-coupling $(q \rightarrow \infty)$ limit the Fano line shape is reduced to a Lorentzian line shape. Interestingly, the Fano asymmetry parameter $(1 /|q|)$ shows strong temperature dependence [see Fig. 2(c)]; it has a high value in the long-range-ordered phase, above $T_{N}(\sim 13 \mathrm{~K})$ it continuously decreases till $\sim 180 \mathrm{~K}$, and thereafter it remains nearly constant up to $330 \mathrm{~K}$ with a quite appreciable value till very high temperature $(\sim 180 \mathrm{~K})$, suggesting the presence of active magnetic excitations far above $T_{N}$. We note that the Fano asymmetry parameter maps nicely onto the dynamic spin susceptibility [discussed later; see Fig. 4(b)], implying that the Fano line shape is also an indicator of spin fractionalization, and the increased value below $200 \mathrm{~K}$ may be translated to a growth of finite spin fractionalization below this temperature. We note that a similar observation has been reported for the other 2D Kitaev materials $\mathrm{RuCl}_{3}$ and $\mathrm{Li}_{2} \mathrm{IrO}_{3}[12,30]$.

We now focus on the temperature evolution of a broad magnetic continuum with spectral weight surviving till $\sim 25 \mathrm{meV}$ [see inset Fig. 1(b), marked by yellow shadings].
In a pure Kitaev material, the underlying quasiparticle excitations in the form of a continuum originates from Majorana fermion scattering [31-33] which survives even in the presence of Heisenberg exchange as a perturbative term [13,19]. In Figs. 3(a) and 3(b) we have shown the temperature evolution of the underlying continuum. To make a quantitative estimate we extracted the intensity of this continuum as a function of temperature [see Fig. 3(c)].

We note that the continuum loses its intensity with decreasing temperature and becomes nearly constant below $\sim 150 \mathrm{~K}$ till $\sim 20 \mathrm{~K}$, and increases upon entering the long-range magnetic ordered phase below $\sim 20 \mathrm{~K}$. Our observation suggests that there is a change in the magnetic scattering below $\sim 150 \mathrm{~K}$, hinting at the development of finite entanglement between spins; also, the existence of this magnetic scattering well above $T_{N}$ is a clear signature of frustrated magnetism. Generally, a broad magnetic continuum evolves into sharp one or two magnon peaks in long-range-ordered AFM systems. However, in $\mathrm{Sm}_{2} \mathrm{ZnIrO}_{6}$, there is no signature of any sharp peak, suggesting that the broad continuum observed here is not a conventional magnetic continuum but does have its origin in an intricate magnetic structure probably dominated by the Kitaev exchange interactions. We note that similar broad magnetic continua have been reported in other systems with QSL as their potential ground state such as herbertsmithite [34], $\mathrm{Cs}_{2} \mathrm{CuCl}_{4}$ [14], $\mathrm{RuCl}_{3}$, and $\mathrm{Li}_{2} \mathrm{IrO}_{3}$ [11-12,30]. In these systems as well, the continuum intensity gains spectral weight as the spin-liquid correlation starts developing well above the long-range magnetic ordering temperature. Keeping in mind 

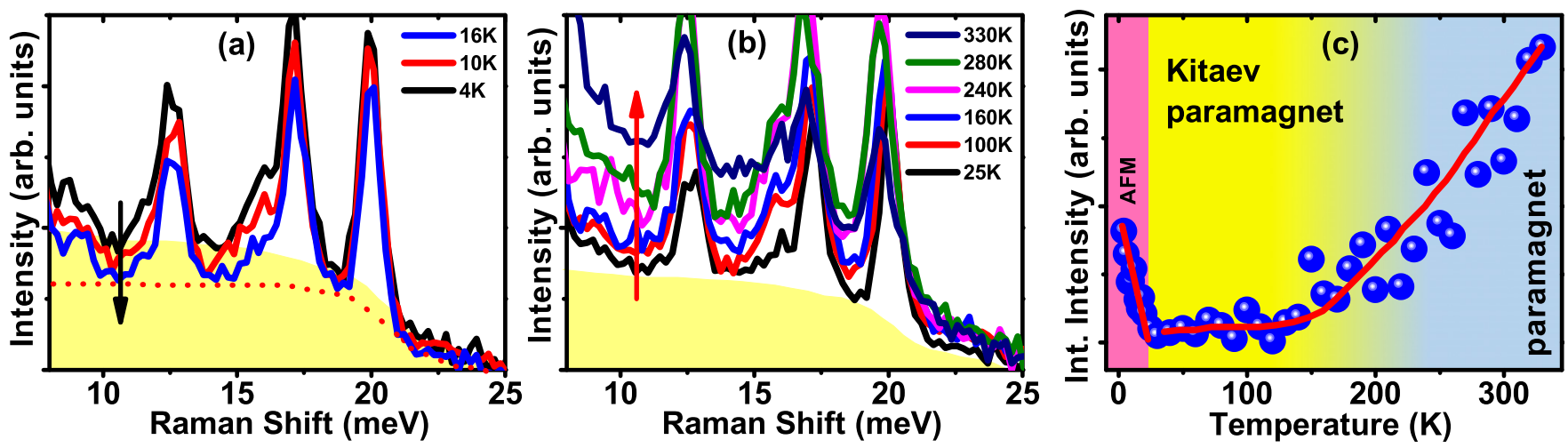

FIG. 3. Magnetic scattering in the double perovskite $\mathrm{Sm}_{2} \mathrm{ZnIrO}_{6}$. (a) Temperature evolution of the background continuum at different temperatures $(4 \mathrm{~K}, 10 \mathrm{~K}$, and $16 \mathrm{~K}$; dotted red line is a guide to eye) and (b) at different temperatures between 25 and $330 \mathrm{~K}$; yellow shading depicts the underlying continuum. (c) Temperature variation of the integrated intensity of the background continuum (solid red line is a guide to the eye and regions of different shading depict different magnetic phases).

that the magnetic continuum in a quasi-2D system extends up to $\sim 3 J_{K}$, we have roughly estimated the Kitaev exchange parameter, $J_{K} \sim 10 \mathrm{meV}$, from the upper cutoff energy of the magnetic continuum by taking the upper range of the magnetic continuum $(\sim 25 \mathrm{meV})$ also as $\sim 3 J_{K}$ in this system. We note that our estimated value of $J_{K}$ is similar to that of $\mathrm{RuCl}_{3}$ $\left(T_{N} \sim 8-14 \mathrm{~K}\right)$ but almost half that of $\mathrm{Li}_{2} \mathrm{IrO}_{3}\left(T_{N} \sim 40 \mathrm{~K}\right)$.

Magnetic Raman scattering has its origin in dynamical spin fluctuations in a quantum paramagnetic phase and may provide a good measure of the thermal fractionalization of quantum spins. To further understand the evolution of this broad magnetic continuum and to identify the Majorana fermion contribution, we have evaluated the dynamic spin susceptibility $\chi^{\text {dyn }}$ associated with the underlying continuum.

The Raman response $\chi^{\prime \prime}(\omega)$ reflects the dynamic properties of collective excitations and is obtained from the raw Raman intensity (intensity $\propto[1+n(\omega)] \chi^{\prime \prime}$ ) simply by dividing it by the Bose thermal factor $[1+n(\omega)]$. To get $\chi^{\text {dyn }}$, first we find Raman conductivity $\chi^{\prime \prime} / \omega$ [see Fig. 4(a) for the temperature evolution] using Raman response. Then using the Kramer-Kronig relation one may obtain the dy- namic spin susceptibility as [35] $\chi^{\mathrm{dyn}}=\lim _{\omega \rightarrow 0} \chi(k=0, \omega) \equiv$ $\frac{2}{\pi} \int_{0}^{\infty} \frac{\chi^{\prime \prime}(\omega)}{\omega} d \omega$; to extract $\chi^{\mathrm{dyn}}, \chi^{\prime \prime} / \omega$ was first extrapolated till $0 \mathrm{meV}$ and integrated up to the upper cutoff value of energy as $25 \mathrm{meV}$, where $\chi^{\prime \prime} / \omega$ shows no change with further increase in the energy. Figure 4(b) shows the temperature evolution of $\chi^{\text {dyn}}$; we note that with decreasing temperature from $330 \mathrm{~K}$, $\chi^{\text {dyn }}$ shows nearly temperature-independent behavior down to $\sim 160-180 \mathrm{~K}$, and upon further lowering the temperature it progressively increases down to $\sim 16 \mathrm{~K}$. Interestingly, below $16 \mathrm{~K}, \chi^{\text {dyn }}$ exhibits an abrupt increase, indicating the appearance of strong spin-spin correlations due to long-range magnetic ordering. As in the pure paramagnetic phase, spins exhibit no correlation; as a result $\chi^{\text {dyn }}$ should be temperature independent. Most interestingly, the increased value of $\chi^{\text {dyn }}$ in the temperature range of $\sim 160 \mathrm{~K}$ to $16 \mathrm{~K}$ indicates the finite entanglement between spins in the paramagnetic state. Interestingly the corresponding energy scale to this temperature $(\sim 160 \mathrm{~K})$ is comparable to the Kitaev exchange interaction $\left(J_{K}\right)$; also around the same temperature, the continuum intensity as well as the Fano asymmetry parameter show an anomaly suggesting that this characteristic temperature
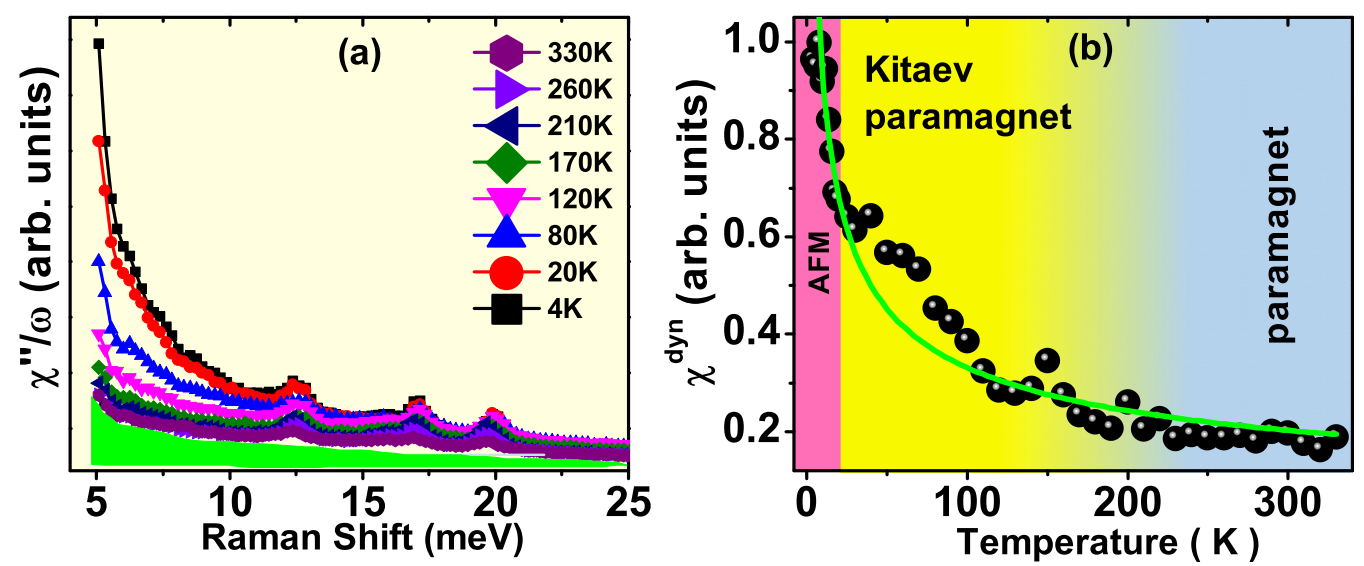

FIG. 4. Raman dynamic response of $\mathrm{Sm}_{2} \mathrm{ZnIrO}_{6}$. (a) Temperature dependence of the Raman conductivity $\chi^{\prime \prime} / \omega$ in the temperature range of 4 to $330 \mathrm{~K}$, and (b) temperature dependence of the dynamic spin susceptibility $\chi^{\text {dyn }}$, extracted by using the Kramer-Kroning relation $\left(\chi^{\mathrm{dyn}} \equiv \frac{2}{\pi} \int_{0}^{25 \mathrm{meV}} \frac{\chi^{\prime \prime}(\omega)}{\omega} d \omega\right)$. Solid green line is the fitted curve with power law $\chi^{\mathrm{dyn}}(T) \propto T^{\beta}(\beta=-0.45)$. Background shading reflects different magnetic phases inferred from the Raman analysis. 
corresponds to a crossover from a paramagnet to quantum paramagnet, at which spins are fractionalized because of the development of a short-range correlation. We also tried to fit the $\chi^{\text {dyn }}$ using a power law as $\chi^{\text {dyn }} \propto T^{\beta}[\beta=-0.45$; see solid green line in Fig. 4(b)]. Unlike a conventional antiferromagnet which shows power-law behavior only below $T_{N}$ and saturates in the paramagnetic phase, $\chi^{\text {dyn }}$ displays a power-law behavior well above the long-range magnetic ordering temperature. The saturation of $\chi^{\text {dyn }}$ is a hallmark of the spin-gas configuration where spins do not talk to each other, i.e., the pure paramagnetic phase; however, the powerlaw dependence of $\chi^{\text {dyn }}$ for the double perovskite $\mathrm{Sm}_{2} \mathrm{ZnIrO}_{6}$ even well above the long-range magnetic ordering temperature reflects the slowly decaying correlation inherent to the spin-liquid phase, and the onset temperature $(\sim 160-180 \mathrm{~K})$ triggers the fractionalization of spins into itinerant spinons [36-37]. This anomaly clearly suggests that the underlying magnetic continuum arises mainly from the fractionalized excitations. We note that our observation of $\mathrm{Sm}_{2} \mathrm{ZnIrO}_{6}$ is analogous to the observation of quasi-Kitaev 2D and 3D honeycomb systems, namely $\mathrm{RuCl}_{3}$ and $\mathrm{Li}_{2} \mathrm{IrO}_{3}$, and similar characteristics of the underlying magnetic continuum in $\mathrm{Sm}_{2} \mathrm{ZnIrO}_{6}$ and these systems indicate that in $\mathrm{Sm}_{2} \mathrm{ZnIrO}_{6}$, Kitaev magnetism may be realized.

In conclusion, our comprehensive Raman scattering study on the double perovskite $\mathrm{Sm}_{2} \mathrm{ZnIrO}_{6}$ evinces the signature of fractionalized excitations. The anomalous evolution of the broad magnetic continuum and Fano asymmetry suggests thermal fractionalization of the spins into fermionic excitations and suggests that the anticipated three-dimensional geometrically frustrated non-honeycomb iridium-based doubleperovskite systems also realize the spin-liquid state. Our results broaden the idea of fractionalized quasiparticles to a non-honeycomb-based 3D geometrically frustrated Kitaev system with Heisenberg exchange as perturbation.

\section{ACKNOWLEDGMENTS}

P.K. thanks the Department of Science and Technology, India, for a grant under the INSPIRE Faculty scheme, and Advanced Material Research Center, IIT Mandi, for the experimental facilities. The authors at Dresden thank Deutsche Forschungsgemeinschaft (DFG) for financial support via Grant No. DFG AS 523/4-1 (S.A.) and via Project No. B01 of SFB 1143 (Project ID 247310070).

\section{APPENDIX}

\section{Experimental and computational details}

Polycrystalline single-phase $\mathrm{Sm}_{2} \mathrm{ZnIrO}_{6}$ samples were synthesized and characterized as described in Ref. [28]. The Raman scattering experiment was done using a Raman spectrometer (LabRAM HR Evolution) in backscattering geometry. A solid-state laser $\lambda=532 \mathrm{~nm}(2.33 \mathrm{eV})$ was used as the excitation source, focused on the sample surface via a $50 \times$ LWD (long working distance) objective lens (0.8 NA). The laser power was kept very low $(\leqslant 1 \mathrm{~mW})$ to avoid any heating effect. The scattered light was dispersed by using a 600 grooves/mm grating, and a Peltier-cooled charge-coupled device (CCD) detector was employed to detect dispersed
TABLE I. Wyckoff positions and irreducible representations of phonon modes of the monoclinic $\left(P 2_{1} / n\right.$; space group No. 14) $\mathrm{Sm}_{2} \mathrm{ZnIrO}_{6}$ double perovskite. $\Gamma_{\text {total }}=12 A_{g}+18 A_{u}+18 B_{u}+$ $12 B_{g}, \Gamma_{\text {Raman }}=12 A_{g}+12 B_{g}$, and $\Gamma_{\text {infrared }}=18 A_{u}+18 B_{u}$.

\begin{tabular}{lcc}
\hline \hline Atom & Wyckoff site & Mode decomposition \\
\hline $\mathrm{Sm}$ & $4 \mathrm{e}$ & $3 A_{g}+3 A_{u}+3 B_{g}+3 B_{u}$ \\
$\mathrm{Zn}$ & $2 \mathrm{a}$ & $3 A_{u}+3 B_{u}$ \\
$\mathrm{Ir}$ & $2 \mathrm{~b}$ & $3 A_{u}+3 B_{u}$ \\
$\mathrm{O}(1)$ & $4 \mathrm{e}$ & $3 A_{g}+3 A_{u}+3 B_{g}+3 B_{u}$ \\
$\mathrm{O}(2)$ & $4 \mathrm{e}$ & $3 A_{g}+3 A_{u}+3 B_{g}+3 B_{u}$ \\
$\mathrm{O}(3)$ & $4 \mathrm{e}$ & $3 A_{g}+3 A_{u}+3 B_{g}+3 B_{u}$ \\
\hline \hline
\end{tabular}

light. Temperature-dependent measurements in the range of 4-330 K were done via a closed-cycle cryostat (Montana Instruments), where the sample was glued on top of the cold finger in an evacuated environment with $\pm 0.1 \mathrm{~K}$ or better temperature accuracy.

The first-principles calculations were performed in the framework of density functional theory (DFT) via employing the plane wave method implemented in the QUANTUM ESPRESSO package [38]. We adopted the generalized gradient approximation (GGA) with the Perdew-BurkeErnzerhof (PBE) exchange-correlation function. The dynamical matrix and phonon frequencies were calculated using density functional perturbation theory [39]. A dense $4 \times 4 \times 4$ Monkhorst-Pack grid was used for numerical integration of the Brillouin zone (BZ) [40]. The plane wave energy cutoff of 35 Ry and charge density cutoff of 350 Ry was used to expand the wave function. The phonon dispersion was calculated with optimized lattice parameters as $a=5.0782 \AA, b=5.5070 \AA$, $c=7.3221 \AA$, and $\beta=89.91^{\circ}$.

\section{Raman scattering and phonon frequency calculations}

For the double perovskite $\mathrm{Sm}_{2} \mathrm{ZnIrO}_{6}$, group theory gives the irreducible representation of the modes as $\Gamma_{\text {Raman }}=$ $12 A_{g}+12 B_{g}$, i.e., a total of 24 Raman active modes; the details are summarized in Table I. Figure 5 shows the unpolarized Raman response $\chi^{\prime \prime}(\omega)$ recorded in a wide energy range of 7-215 $\mathrm{meV}$ at $4 \mathrm{~K}$, showing both first and second order phonon modes. The Raman response exhibits a total of 23 modes, labeled as S1-S23. The spectrum is fitted with a sum of Lorentzian functions to extract a quantitative value of phonon frequency $(\omega)$ and linewidth $(\Gamma)$.

Furthermore, we performed zone-centered $(q=0,0,0)$ phonon frequency calculations to have insight on the specific symmetry associated with a particular phonon mode and its symmetry assignment. According to our calculations phonon modes S1-S18 are attributed to being first-order phonon modes; however, high-frequency modes S19-S23 are secondorder modes.

Figures 6(a) and 6(b) illustrate the calculated phonon dispersion curves along the high-symmetry directions and the total phonon density of states (DOS) of $\mathrm{Sm}_{2} \mathrm{ZnIrO}_{6}$. The phonon dispersion curves show no imaginary frequencies, indicating the dynamic stability of this compound. The schematic representation of the calculated eigenvectors for zone-centered phonon modes observed experimentally is 


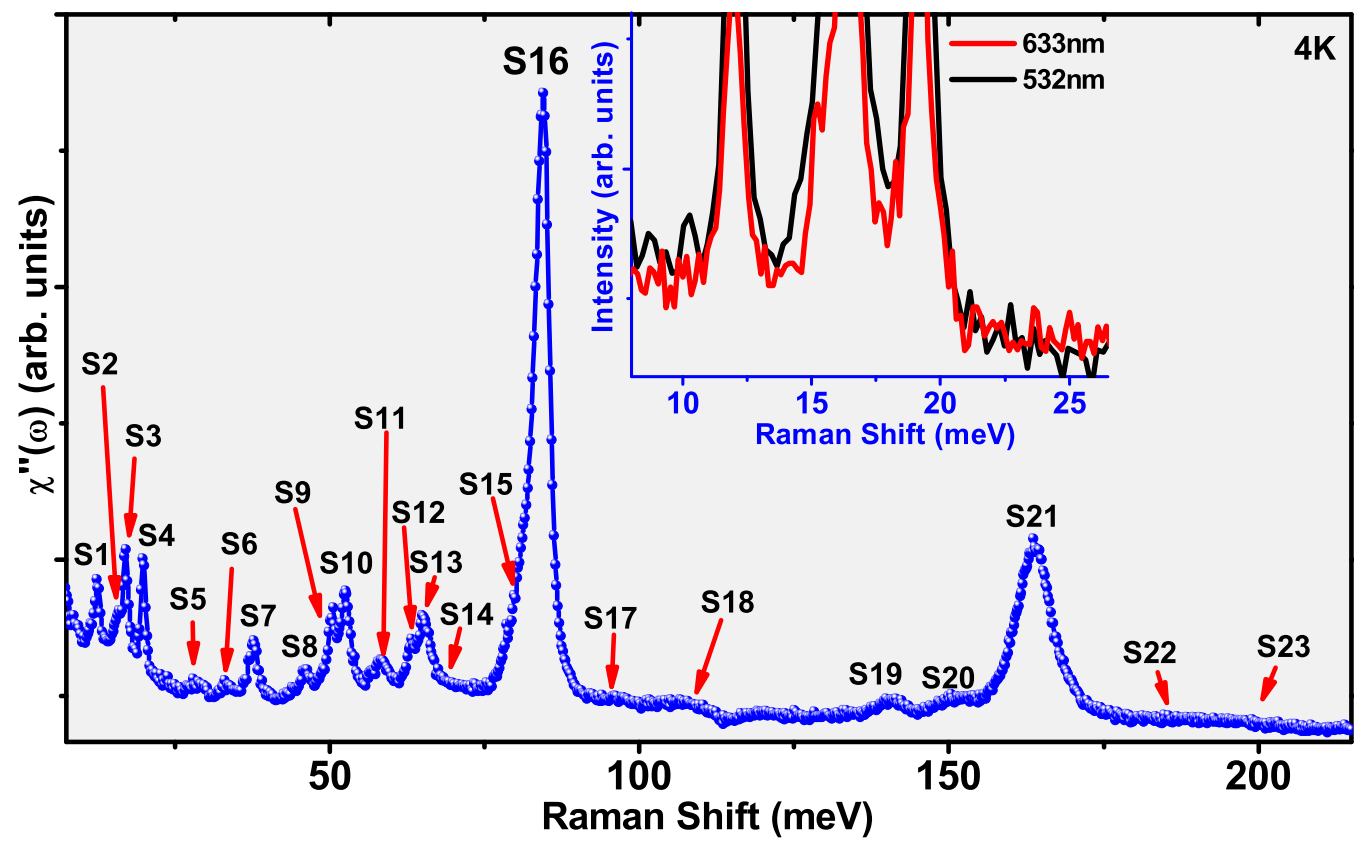

FIG. 5. Raman response $\chi^{\prime \prime}(\omega)$ of the double perovskite $\mathrm{Sm}_{2} \mathrm{ZnIrO}_{6}$ recorded at $4 \mathrm{~K}$ in a wide energy range of 7-215 meV. Inset shows raw Raman data recorded at room temperature with $532 \mathrm{~nm}(2.33 \mathrm{eV})$ and $633 \mathrm{~nm}(1.96 \mathrm{eV})$ excitation laser.

shown in Fig. 7. The phonon modes below $250 \mathrm{~cm}^{-1}$ (S1-S5) are mainly attributed to the displacement of heavy rare-earth $\left(\mathrm{Sm}^{3+}\right.$ motions) ions. Above $250 \mathrm{~cm}^{-1}$ all the phonon modes are characterized by the motion of oxygen atoms associated with $\mathrm{Ir} / \mathrm{ZnO}_{6}$ octahedra. The assignment of experimentally observed phonon modes is done in accordance with our lattice dynamics calculations listed in Table II.

\section{Temperature dependence of the phonon modes}

Figures 8 and 9 illustrate the mode frequency and linewidth of the prominent first as well as second order phonon modes as a function of temperature. The following observations can be made: (i) The phonon modes are observed to show an anomalous softening of $\sim 1.5 \%$ in low-frequency modes (S1-S3) and $\sim 0.5 \%$ in high-frequency modes (S7, S9, S10, and S13) in the low-temperature region with respect to their value at $16 \mathrm{~K}$. (ii) Furthermore, we see substantial line broadening (increase in phonon lifetime) of phonon modes, $\sim 10 \%$ and $\sim 5 \%$ for low-frequency modes (S1 and S3) and high-frequency modes (S9 and S10), respectively, below $\sim 16 \mathrm{~K}$ with decrease in temperature. (iii) Interestingly, we note a clear and sharp discontinuity at $\sim 180 \mathrm{~K}$, phonon hardening $\sim 2 \mathrm{~cm}^{-1}$ in the $\mathrm{S} 1, \mathrm{~S} 3$, and S7 modes with decrease in temperature. This discontinuity in phonon mode frequencies may occur because of local structural changes within the crystal or spin reorientations around this temperature.

To quantify the temperature dependence of phonon frequencies and the damping constant, we have fitted the mode
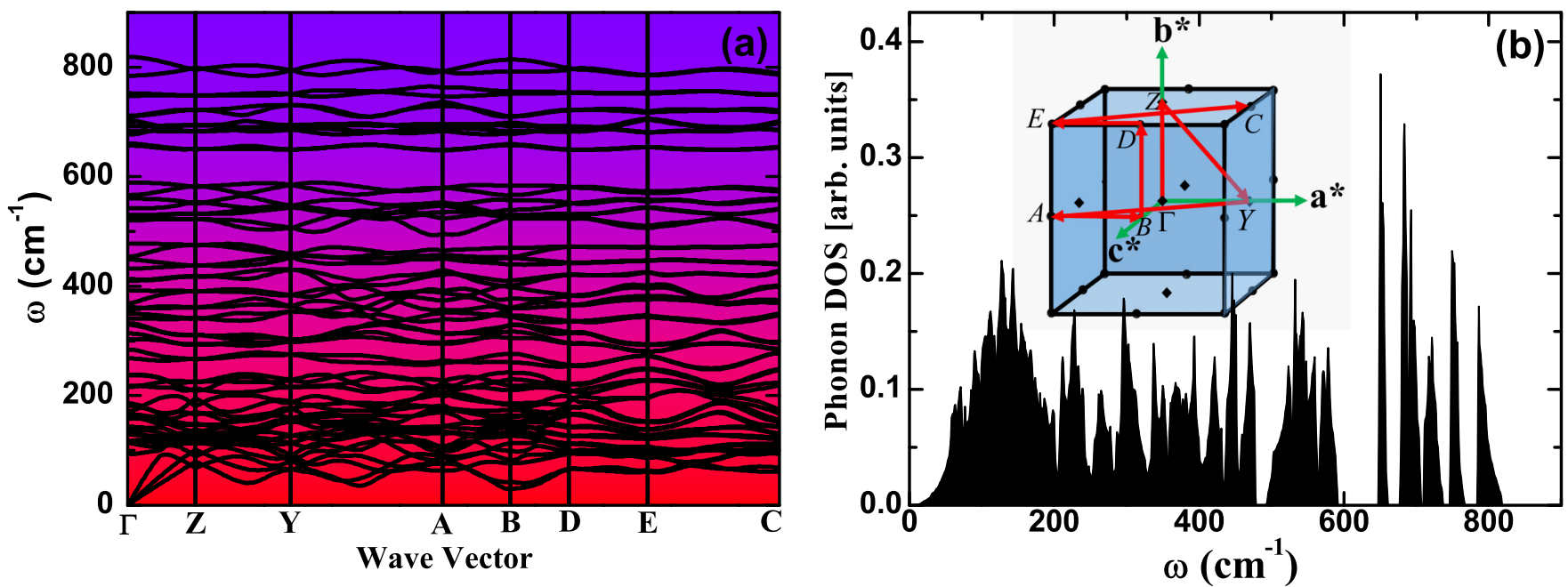

FIG. 6. Calculated phonon dispersion curves along high-symmetry directions (a), and (b) total phonon density of states of $\mathrm{Sm}_{2} \mathrm{ZnIrO}_{6}$ (inset: first Brillion zone with high-symmetry directions in red). 


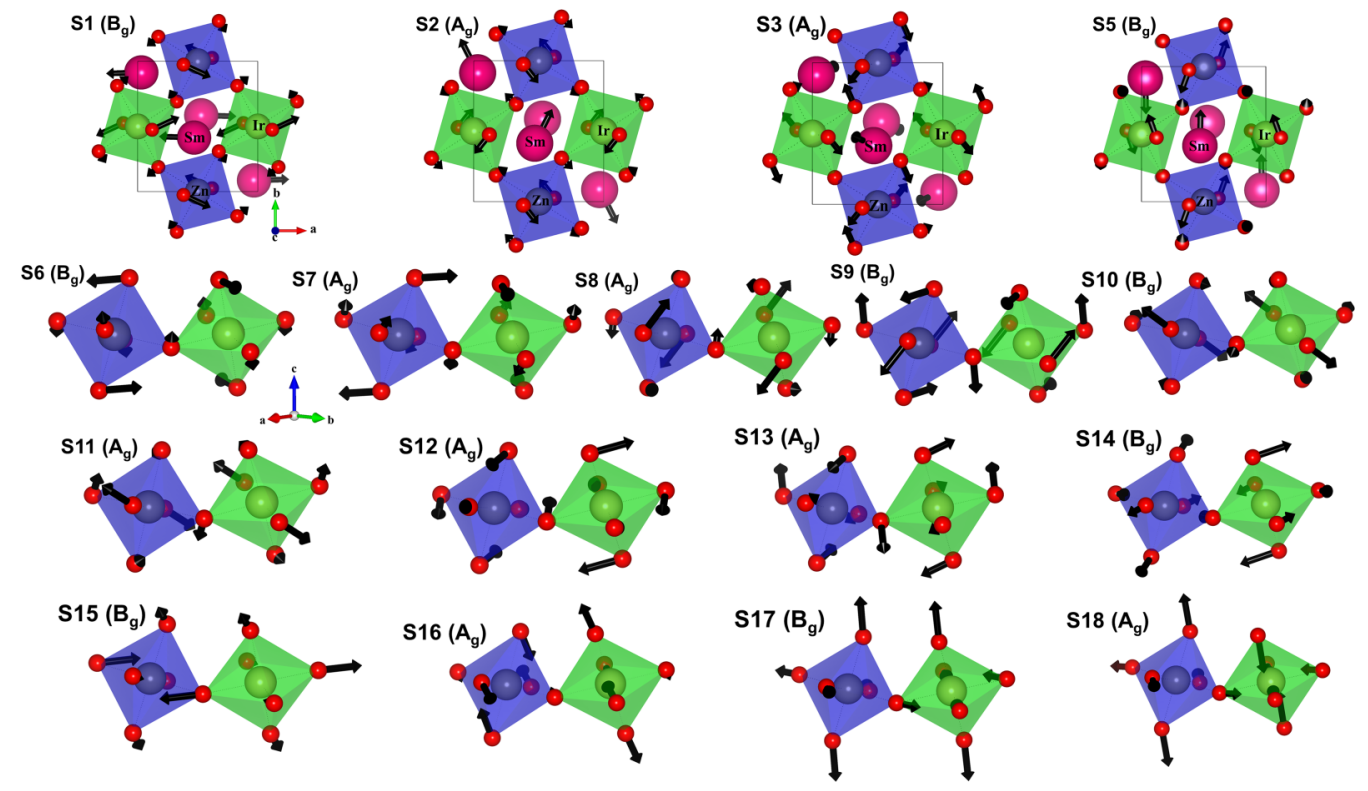

FIG. 7. Calculated eigenvectors for all the experimentally detected (first-order) phonon modes. Pink, gray, dark yellow, and red spheres represent $\mathrm{Sm}, \mathrm{Zn}$, Ir, and oxygen atoms, respectively. Black arrows on the atoms represent the direction of characteristic displacement, and the size of the arrow represents the magnitude of vibration; $a, b$, and $c$ represent the crystallographic axes.

TABLE II. List of the experimentally observed phonon mode frequencies at $4 \mathrm{~K}$, fitting parameters (fitted using equations as described in the text), and DFT-calculated zone-centered frequencies. Mode assignment (with displacement of atoms involved) has been done via our DFTbased calculations. Low-frequency modes S1-S5 $\left(<250 \mathrm{~cm}^{-1}\right)$ are associated mainly with the vibrations of Sm atoms and small contributions from the vibration of oxygen atoms; modes above $\sim 250 \mathrm{~cm}^{-1}(\mathrm{~S} 6-\mathrm{S} 18)$ are associated with the vibration of oxygen atoms within the octahedral unit $\mathrm{Zn} / \mathrm{IrO}_{6}$. Calculated frequencies that are not observed experimentally (units are in $\mathrm{cm}^{-1}$ ) are $167.7\left(B_{g}\right), 332.1\left(B_{g}\right), 349.6\left(A_{g}\right), 529.5\left(B_{g}\right)$, $657.4\left(A_{g}\right)$, and $703.4\left(B_{g}\right)$.

\begin{tabular}{|c|c|c|c|c|c|c|c|c|}
\hline \multirow[b]{2}{*}{ Mode assignment } & \multirow[b]{2}{*}{ Expt. $\omega(4 \mathrm{~K})$} & \multirow[b]{2}{*}{ DFT $\omega$} & \multicolumn{6}{|c|}{ Fitted parameters } \\
\hline & & & $\omega_{0}$ & $A$ & $B$ & $\Gamma_{0}$ & $C$ & $D$ \\
\hline $\mathrm{S} 1-B_{g}(\mathrm{Sm}, \mathrm{O})$ & 100.9 & 114.6 & $102.1 \pm 0.3$ & $-0.09 \pm 0.1$ & $-0.005 \pm 0.002$ & $8.7 \pm 0.3$ & $-0.27 \pm 0.14$ & $0.002 \pm 0.001$ \\
\hline $\mathrm{S} 2-A_{g}(\mathrm{Sm}, \mathrm{O})$ & 126.9 & 117.2 & $128.2 \pm 0.3$ & $-0.22 \pm 0.1$ & $-0.003 \pm 0.001$ & $10.2 \pm 1.0$ & $-0.37 \pm 0.03$ & $-0.004 \pm 0.001$ \\
\hline $\mathrm{S} 3-A_{g}(\mathrm{Sm}, \mathrm{O})$ & 137.9 & 161.6 & $139.1 \pm 0.2$ & $-0.32 \pm 0.1$ & $-0.008 \pm 0.002$ & $6.7 \pm 0.5$ & $0.21 \pm 0.1$ & $0.01 \pm 0.007$ \\
\hline $\mathrm{S} 4-A_{g}(\mathrm{Sm}, \mathrm{O})$ & 160.9 & 165.6 & $162.1 \pm 0.3$ & $-0.49 \pm 0.15$ & $-0.01 \pm 0.003$ & $6.2 \pm 0.2$ & $0.38 \pm 0.2$ & $0.003 \pm 0.001$ \\
\hline $\mathrm{S} 5-B_{g}(\mathrm{Sm}, \mathrm{O})$ & 231.5 & 185.8 & $229.9 \pm 1.8$ & $-0.12 \pm 0.01$ & $-0.005 \pm 0.002$ & $19.6 \pm 3.4$ & $1.5 \pm 0.6$ & $-0.05 \pm 0.02$ \\
\hline $\mathrm{S} 6-A_{g}(\mathrm{O})$ & 269.6 & 302.2 & $270.5 \pm 1.7$ & $0.54 \pm 0.3$ & $-0.14 \pm 0.04$ & $17.4 \pm 2.9$ & $2.8 \pm 0.7$ & $0.23 \pm 0.07$ \\
\hline $\mathrm{S} 7-B_{g}(\mathrm{O})$ & 304.4 & 313.7 & $307.9 \pm 0.6$ & $-2.4 \pm 0.5$ & $-0.03 \pm 0.01$ & $10.1 \pm 1.1$ & $3.5 \pm 0.9$ & $-0.06 \pm 0.003$ \\
\hline $\mathrm{S} 8-A_{g}(\mathrm{O})$ & 370.5 & 362.1 & $376.9 \pm 1.5$ & $-5.1 \pm 1.2$ & $0.1 \pm 0.04$ & $17.1 \pm 3.7$ & $2.29 \pm 0.7$ & $-0.19 \pm 0.02$ \\
\hline $\mathrm{S} 9-B_{g}(\mathrm{O})$ & 406.6 & 409.2 & $410.2 \pm 1.1$ & $-3.26 \pm 1.2$ & $-0.05 \pm 0.01$ & $10.5 \pm 2.9$ & $0.69 \pm 0.2$ & $0.9 \pm 0.20$ \\
\hline $\mathrm{S} 10-B_{g}(\mathrm{O})$ & 424.6 & 431.8 & $429.2 \pm 0.9$ & $-4.3 \pm 1.0$ & $0.07 \pm 0.02$ & $10.1 \pm 2.5$ & $4.95 \pm 2.6$ & $-0.04 \pm 0.01$ \\
\hline $\mathrm{S} 11-A_{g}(\mathrm{O})$ & 470.5 & 453.6 & $478.1 \pm 02.3$ & $-5.9 \pm 2.4$ & $-0.02 \pm 0.001$ & $29.6 \pm 5.3$ & $1.7 \pm 0.8$ & $0.6 \pm 0.10$ \\
\hline $\mathrm{S} 12-A_{g}(\mathrm{O})$ & 509.0 & 519.3 & $511.9 \pm 1.9$ & $-2.08 \pm 1.6$ & $0.10 \pm 0.03$ & $5.3 \pm 1.6$ & $2.5 \pm 0.7$ & $1.7 \pm 0.80$ \\
\hline $\mathrm{S} 13-A_{g}(\mathrm{O})$ & 526.9 & 525.8 & $533.7 \pm 2.7$ & $-6.1 \pm 2.6$ & $-0.06 \pm 0.02$ & $16.7 \pm 4.9$ & $3.6 \pm 1.6$ & $-0.5 \pm 0.20$ \\
\hline $\mathrm{S} 14-B_{g}(\mathrm{O})$ & 564.9 & 559.7 & & & & & & \\
\hline $\mathrm{S} 15-B_{g}(\mathrm{O})$ & 662.9 & 661.2 & $667.8 \pm 5.2$ & $-5.9 \pm 1.3$ & $-0.9 \pm 0.04$ & $22.2 \pm 3.8$ & $16.8 \pm 4.7$ & $-0.9 \pm 0.30$ \\
\hline $\mathrm{S} 16-A_{g}(\mathrm{O})$ & 681.3 & 692.6 & $692.7 \pm 4.6$ & $-10.9 \pm 0.2$ & $-0.4 \pm 0.12$ & $5.3 \pm 2.1$ & $13.8 \pm 3.9$ & $-0.08 \pm 0.03$ \\
\hline $\mathrm{S} 17-B_{g}(\mathrm{O})$ & 785.6 & 784.5 & & & & & & \\
\hline $\mathrm{S} 18-A_{g}(\mathrm{O})$ & 859.4 & 818.7 & & & & & & \\
\hline S19 & 1131.9 & & & & & & & \\
\hline S20 & 1215.1 & & & & & & & \\
\hline $\mathrm{S} 21$ & 1321.2 & & & & & & & \\
\hline S22 & 1465.7 & & & & & & & \\
\hline S23 & 1558.7 & & & & & & & \\
\hline
\end{tabular}



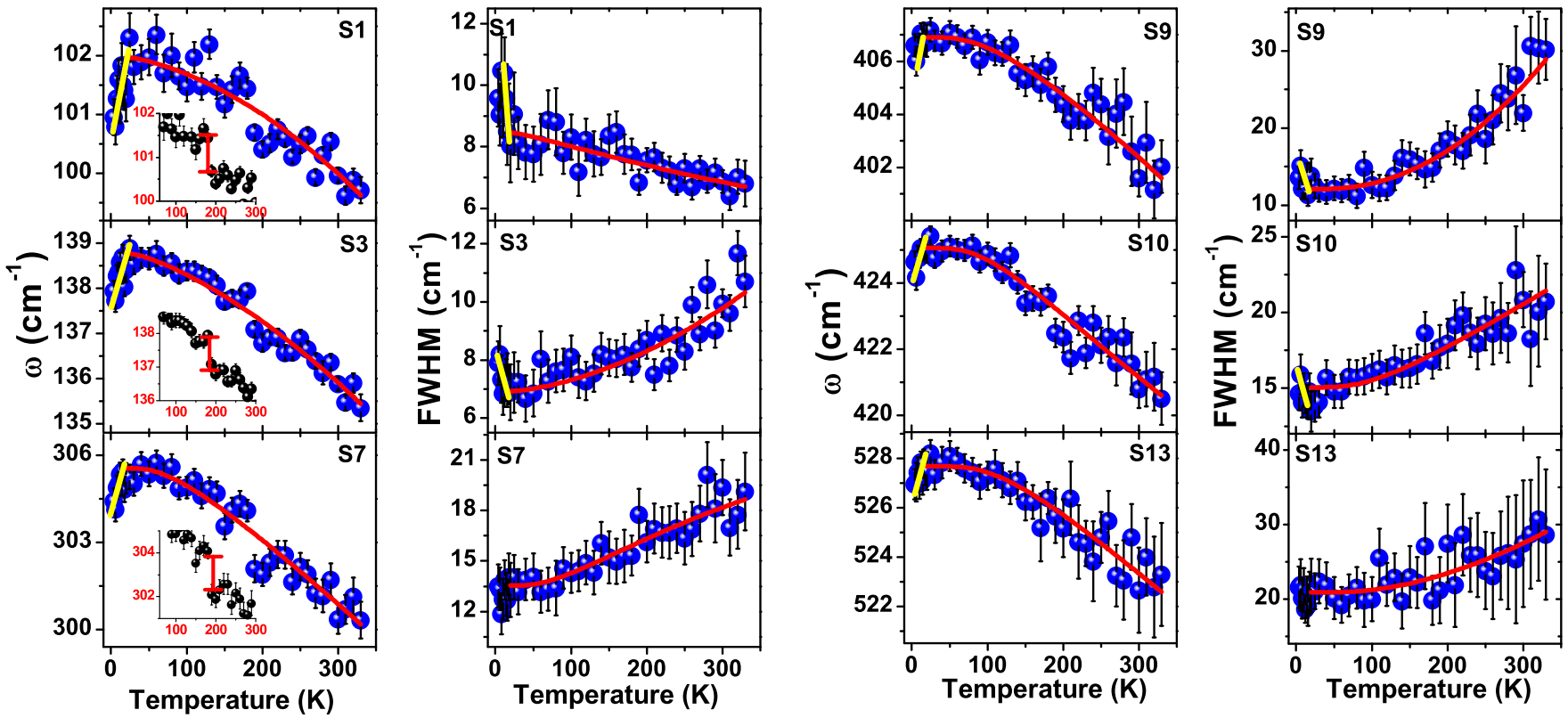

FIG. 8. Temperature variation of mode frequency and linewidth of S1, S3, S7, S9, S10, and S13 modes. Solid red lines are fitted curves as described in the text, and yellow lines are guides to the eye. Inset of S1, S3, and S7 shows zoomed view of frequency in temperature range of $60 \mathrm{~K}$ to $300 \mathrm{~K}$, and solid red vertical line is a guide to eye.

frequency and linewidth of the first-order phonon modes with an anharmonic phonon-phonon interaction model in the temperature range of $\sim 20-330 \mathrm{~K}$. As within the harmonic approximation, the phonon frequencies do not exhibit any temperature dependence and the corresponding phonon lifetime is infinite (zero linewidth). In the real system the change in phonon frequencies and finite phonon lifetime arises due to phonon-phonon interaction (i.e., creation and annihilation of phonons); consequently we need to take into account the anharmonic contributions in the potential term as $U_{\mathrm{anh}}(r)=g r^{3}+m r^{4}+\cdots \equiv \beta\left(a^{+} a^{+} a+\right.$ $\left.a^{+} a a\right)+\gamma\left(a^{+} a^{+} a^{+} a+a^{+} a^{+} a a\right)+\cdots$, where $a^{+}$and $a$ are the phonon creation and annihilation operators, respectively. In the anharmonic interaction model fit we have taken both cubic (three-phonon interactions, i.e., cubic anharmonicity; a decay into two acoustic phonons of equal frequency) and quadratic (four-phonon interactions, i.e., quadratic anharmonicity; a decay into three acoustic phonons of equal frequency) terms given as [41]

$$
\omega(T)=\omega_{0}+A\left(1+\frac{2}{e^{x}-1}\right)+B\left(1+\frac{3}{e^{y}-1}+\frac{3}{\left(e^{y}-1\right)^{2}}\right)
$$

and

$\Gamma(T)=\Gamma_{0}+C\left(1+\frac{2}{e^{x}-1}\right)+D\left(1+\frac{3}{e^{y}-1}+\frac{3}{\left(e^{y}-1\right)^{2}}\right)$,

where $\omega_{0}$ and $\Gamma_{0}$ are the mode frequency and linewidth at absolute-zero temperature, respectively, $x=\frac{\hbar \omega_{0}}{2 k_{B} T}, y=\frac{\hbar \omega_{0}}{3 k_{B} T}$, and $A, B, C$, and $D$ are constant. The solid red lines in Fig. 8 are fits of the frequency and linewidth to the anharmonic interaction model in the temperature range of $20-330 \mathrm{~K}$. The temperature variation of phonon modes in this range can be well explained within the cubic and quartic anharmonicities. The values obtained from fitting are summarized in Table II. It is worth noting that from the fitted values the cubic
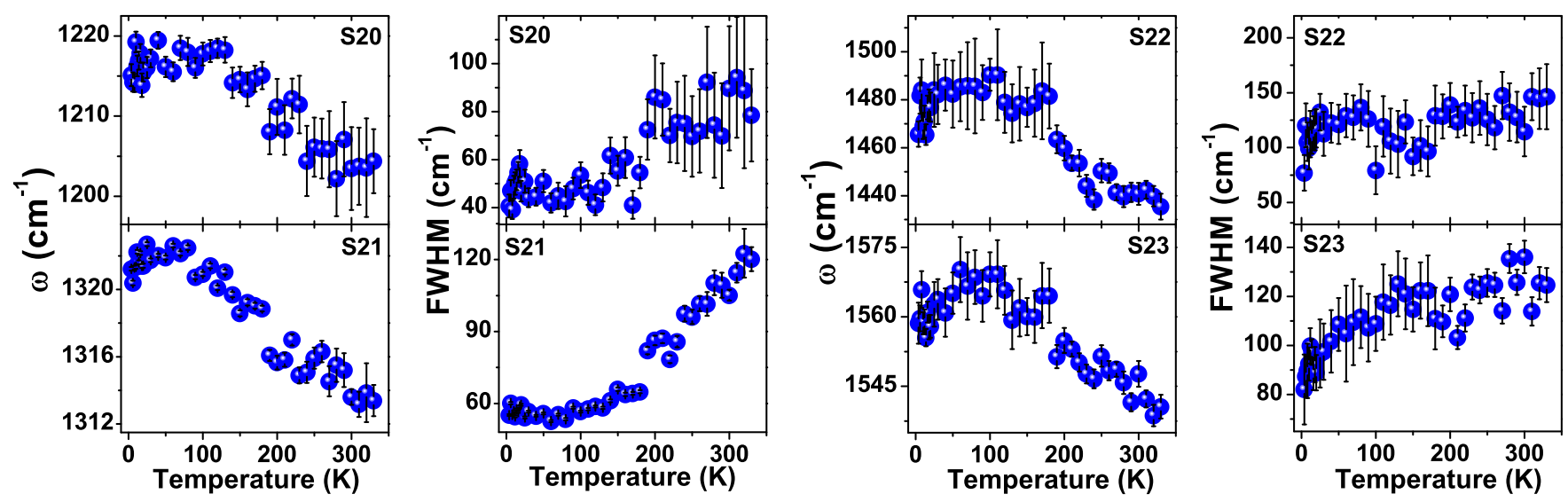

FIG. 9. Temperature variation of mode frequency and linewidth of the second-order phonon modes S20, S21, S22, and S23. 
anharmonicity decay is primarily responsible for the temperature dependences of phonon modes. The deviation of mode frequencies and linewidth from the curve estimated by the anharmonic interaction model is observed in the lowtemperature regime below $\sim 20 \mathrm{~K}$. The phonon renormalization in the low temperature could be due to additional decay channels, namely, the interaction of phonons with other quasiparticle excitations. As at low temperature the effect of phonon-phonon interaction on the self-energy parameters is minimal. Therefore, the renormalization of phonon frequency below the magnetic-ordering temperature can be understood due to the coupling of the lattice with magnetic degrees of freedom through spin-phonon coupling. The spinphonon coupling Hamiltonian can be written as [42] $H=$ $\frac{1}{2} \sum_{i j} J_{i j}\left(\vec{S}_{i} \cdot \vec{S}_{j}\right)$, where $\vec{S}_{i} \cdot \vec{S}_{j}$ is the spin-spin correlation function and the index $(i, j)$ runs through the lattice; $J_{i j}$ is the superexchange integral. The strength of the spin-phonon cou- pling of a particular phonon mode depends on the amplitude of vibration (atomic displacement) associated with that mode and leads to modulation of the exchange coupling. Therefore, the amount of renormalization may be different for different phonon modes. The phonon frequency is related to the spinspin interaction by the relation given as $[15,43,44] \omega \approx \omega_{0}^{\mathrm{ph}}+$ $\lambda\left\langle\vec{S}_{i} \cdot \vec{S}_{j}\right\rangle$, where $\omega$ and $\omega_{0}^{\mathrm{ph}}$ correspond to the phonon mode frequency and bare phonon frequency (without spin-phonon coupling), $\lambda=\frac{\partial^{2} J_{i j}}{\partial u^{2}}$ is the spin-phonon coupling coefficient ( $u$ is the atomic displacement), and $\left\langle\vec{S}_{i} \cdot \vec{S}_{j}\right\rangle$ denotes the spinspin correlation between the $i$ th and $j$ th magnetic ions. The coupling of the lattice with the underlying magnetic degrees of freedom occurs because of modulation of the exchange integral $J_{i j}$ between the magnetic ions in the long-rangeordered phase, resulting in significant renormalization of the phonon self-energy parameters.
[1] B. J. Kim, H. Jin et al., Phys. Rev. Lett. 101, 076402 (2008).

[2] F. Wang and T. Senthil, Phys. Rev. Lett. 106, 136402 (2011).

[3] B. J. Yang and Y. B. Kim, Phys. Rev. B 82, 085111 (2010).

[4] C. H. Kim, H. S. Kim, H. Jeong, H. Jin, and J. Yu, Phys. Rev. Lett. 108, 106401 (2012).

[5] L. Balents, Nature (London) 464, 199 (2010).

[6] A. Kitaev, Ann. Phys. 321, 2 (2006).

[7] G. Jackeli and G. Khaliullin, Phys. Rev. Lett. 102, 017205 (2009).

[8] S. K. Choi, R. Coldea et al., Phys. Rev. Lett. 108, 127204 (2012).

[9] X. Liu, T. Berlijn et al., Phys. Rev. B 83, 220403(R) (2011).

[10] K. W. Plumb, J. P. Clancy, L. J. Sandilands, V. V. Shankar, Y. F. Hu, K. S. Burch, H.-Y. Kee, and Y.-J. Kim, Phys. Rev. B 90, 041112(R) (2014).

[11] L. J. Sandilands, Y. Tian, K. W. Plumb, Y.-J. Kim, and K. S. Burch, Phys. Rev. Lett. 114, 147201 (2015).

[12] A. Glamazda, P. Lemmens, S.-H. Do, Y. S. Kwon, and K.-Y. Choi, Phys. Rev. B 95, 174429 (2017).

[13] A. Banerjee, C. A. Bridges et al., Nat. Mater. 15, 733 (2016).

[14] R. Coldea, D. A. Tennant, and Z. Tylczynski, Phys. Rev. B 68, 134424 (2003).

[15] P. Kumar, A. Bera et al., Phys. Rev. B 85, 134449 (2012).

[16] I. Kimchi and A. Vishwanath, Phys. Rev. B 89, 014414 (2014).

[17] A. M. Cook, S. Matern, C. Hickey, A. A. Aczel, and A. Paramekanti, Phys. Rev. B 92, 020417(R) (2015).

[18] G. Cao, A. Subedi et al., Phys. Rev. B 87, 155136 (2013).

[19] A. A. Aczel, A. M. Cook, T. J. Williams, S. Calder, A. D. Christianson, G.-X. Cao, D. Mandrus, Y.-B. Kim, and A. Paramekanti, Phys. Rev. B 93, 214426 (2016).

[20] A. A. Aczel, J. P. Clancy et al., Phys. Rev. B 99, 134417 (2019).

[21] A. Revelli, C. C. Loo et al., Phys. Rev. B 100, 085139 (2019).

[22] B. Perreault, J. Knolle, N. B. Perkins, and F. J. Burnell, Phys. Rev. B 92, 094439 (2015).

[23] W. Hayes and R. Loudon, Scattering of Light by Crystals (Dover Books, 2004).

[24] P. Kumar, A. Bera, D. V. S Muthu, P. M. Shirage, A. Iyo, and A. K. Sood, Appl. Phys. Lett. 100, 222602 (2012).
[25] B. Singh, G. A. Cansever, T. Dey, A. Maljuk, S. Wurmehl, B. Büchner, and P. Kumar, J. Phys.: Condens. Matter 31, 065603 (2019).

[26] P. Kumar, D. V. S. Muthu, L. Harnagea, S. Wurmehl, B. Büchner, and A. K. Sood, J. Phys.: Condens. Matter 26, 305403 (2014).

[27] P. Lemmens, G. Guntherodt, and C. Gros, Phys. Rep. 375, 1 (2003).

[28] M. Vogl et al., arXiv:1910.13552.

[29] U. Fano, Phys. Rev. 124, 1866 (1961).

[30] A. Glamazda, P. Lemmens, S.-H. Do, Y. S. Choi, and K.-Y. Choi, Nat. Commun. 7, 12286 (2016).

[31] G. Baskaran, S. Mandal, and R. Shankar, Phys. Rev. Lett. 98, 247201 (2007).

[32] J. Knolle, G.-W. Chern, D. L. Kovrizhin, R. Moessner, and N. B. Perkins, Phys. Rev. Lett. 113, 187201 (2014).

[33] J. Nasu, J. Knolle, D. L. Kovrizhin, Y. Motome, and R. Moessner, Nat. Phys. 12, 912 (2016).

[34] D. Wulferding, P. Lemmens, P. Scheib, J. Röder, P. Mendels, S. Chu, T. Han, and Y. S. Lee, Phys. Rev. B 82, 144412 (2010).

[35] Y. Gallais and I. Paul, C. R. Phys. 17, 113 (2016).

[36] M. Hermele, Y. Ran, P. A. Lee, and X.-G. Wen, Phys. Rev. B 77, 224413 (2008).

[37] J. Nasu, M. Udagawa, and Y. Motome, Phys. Rev. Lett. 113, 197205 (2014).

[38] P. Giannozzi, S. Baroni et al., J. Phys.: Condens. Matter 21, 395502 (2009).

[39] J. P. Perdew, A. Ruzsinszky et al., Phys. Rev. Lett. 100, 136406 (2008).

[40] P. Giannozzi, S. de Gironcoli, P. Pavone, and S. Baroni, Phys. Rev. B 43, 7231 (1991).

[41] M. Balkanski, R. F. Wallis, and E. Haro, Phys. Rev. B 28, 1928 (1983).

[42] E. Granado, A. García, J. A. Sanjurjo, C. Rettori, I. Torriani, F. Prado, R. D. Sánchez, A. Caneiro, and S. B. Oseroff, Phys. Rev. B 60, 11879 (1999).

[43] D. J. Lockwood and M. G. Cottam, J. Appl. Phys. 64, 5876 (1988).

[44] E. Granado, N. O. Moreno, H. Martinho, A. García, J. A. Sanjurjo, I. Torriani, C. Rettori, J. J. Neumeier, and S. B. Oseroff, Phys. Rev. Lett. 86, 5385 (2001). 